\title{
Fluocinolone Acetonide Intravitreal Implant for Treating Recurrent Non-infectious Uveitis: An Evidence Review Group Perspective of a NICE Single Technology Appraisal
}

\author{
Xavier G. L. V. Pouwels ${ }^{1} @$. Svenja Petersohn ${ }^{1} \cdot$ Vanesa Huertas Carrera $^{2} \cdot$ Alastair K. Denniston $^{3,4} \cdot$ Annette Chalker $^{2}$. \\ Heike Raatz ${ }^{2,5} \cdot$ Nigel Armstrong $^{2} \cdot$ Dhwani Shah $^{2} \cdot$ Willem Witlox $^{1} \cdot$ Gill Worthy $^{2} \cdot$ Caro Noake $^{2} \cdot$ Rob Riemsma $^{2}$. \\ Jos Kleijnen $^{2,6}$. Manuela A. Joore ${ }^{1,6}$
}

Published online: 8 November 2019

(c) The Author(s) 2019

\begin{abstract}
The National Institute for Health and Care Excellence (NICE) invited Alimera Sciences, the company manufacturing fluocinolone acetonide intravitreal implant (FAc) $0.19 \mathrm{mg}$ (tradename ILUVIEN ${ }^{\circledR}$ ), to submit evidence on the clinical and costeffectiveness of FAc for treating recurrent non-infectious uveitis. Kleijnen Systematic Reviews Ltd, in collaboration with Maastricht University Medical Centre +, was commissioned to act as the independent Evidence Review Group (ERG). This paper contains a summary of the clinical and cost-effectiveness evidence submitted by the company, the ERG's critique on the submitted evidence, and the guidance issued by the NICE Appraisal Committee (AC). The company submission (CS) was mainly informed by the PSV-FAI-001 trial in which FAc was compared with (limited) current practice [(L)CP], which was not considered to be representative of UK clinical practice by the ERG. There was no comparison of FAc to any treatment listed in the final scope, and especially to the dexamethasone intravitreal implant (dexamethasone), which was considered to be a relevant comparator by the AC. The primary outcome of the PSV-FAI-001 was recurrence of uveitis in the treated eye. Most of the events for the primary outcome were imputed during the PSV-FAI-001 trial, which probably led to an overestimation of the number of recurrences of disease, and a biased estimate of the relative effectiveness of FAc versus (L)CP. Finally, the place of FAc in the treatment pathway was not clearly defined by the company. Substantial uncertainty surrounded the cost-effectiveness results due to the shortcomings of the clinical evidence. Additionally, the quality of life of patients was not measured during the PSV-FAI-001 trial and long-term effectiveness data of FAc were lacking. The ERG adjusted several issues identified in the CS and added dexamethasone as a comparator in the decision analytic model. The ERG presented multiple analyses as base-cases because several elements of the assessment remained uncertain. The fully incremental ERG results ranged from dexamethasone (extendedly) dominating FAc (when assuming a hazard ratio of 1 or 0.7 for dexamethasone versus FAc) to an incremental cost-effectiveness ratio (ICER) of $£ 30,153$ per quality-adjusted lifeyear (QALY) gained for FAc versus (L)CP [when assuming a hazard ratio of 0.456 for dexamethasone versus (L)CP]. The ICER of FAc versus (L)CP ranged from $£ 12,325$ to $£ 30,153$ per QALY gained. After a second AC meeting where alternative company scenarios comparing FAc with dexamethasone were considered by the AC, the AC concluded that "the results of the company's analyses ranged from the fluocinolone acetonide implant being dominant (that is, it was more effective and costs less), to an ICER of $£ 29,461$ per QALY gained, and most of the ICERs were below $£ 20,000$ per QALY gained". Therefore, the AC recommended FAc as a cost-effective use of National Health Service (NHS) resources for treating recurrent non-infectious uveitis affecting the posterior segment of the eye in the final TA590 guidance (published July 2019).
\end{abstract}

\section{Introduction}

As part of the single technology appraisal (STA) process, the National Institute for Health and Care Excellence (NICE) invited Alimera Sciences to submit evidence regarding the

Extended author information available on the last page of the article clinical and cost-effectiveness of fluocinolone acetonide intravitreal implant (FAc) for the treatment of recurrent noninfectious uveitis. NICE is an independent organisation that provides national guidance in the UK on promoting good health and preventing and treating ill health in priority areas with significant impact. To be recommended by NICE for use within the UK National Health Service (NHS), health 


\section{Key Points for Decision Makers}

Fluocinolone acetonide intravitreal implant (FAc) has been recommended within its marketing authorisation, while the evidence submitted did not include any relevant comparators and was supported by a likely biased effectiveness estimation.

The clinical trial results for the FAc intravitreal implant compared with (limited) current practice were difficult to interpret and were very uncertain.

The benefit-risk ratio for FAc (when compared with no treatment) seemed good. However, the effectiveness of FAc compared with active comparators was unclear due to the imputation methods and the comparator used in the trial. This uncertainty was not propagated into the probabilistic sensitivity analysis, but the impact of that uncertainty on the results was extensively explored by the Evidence Review Group through scenario analyses.

technologies must represent a clinically and cost-effective use of the NHS resources. During the STA process, the company submits a company submission (CS) [1], which is reviewed and criticised by an independent academic organisation, the Evidence Review Group (ERG). This ERG critique is compiled in the ERG report. Both the CS and the ERG report are then considered by an Appraisal Committee (AC), which issues an Appraisal Consultation Document (ACD) indicating the initial guidance regarding the use of the technology. The ACD may be commented upon by stakeholders. These comments may lead to a subsequent ACD or a Final Appraisal Determination (FAD) [2], which is open to appeal. In this case, there was no appeal. The final TA590 guidance was published in July 2019.

This paper presents a summary of the ERG report, delivered by Kleijnen Systematic Reviews Ltd, in collaboration with Maastricht University Medical Centre + [3], and the development of the NICE guidance. It highlights key methodological issues emerging from the appraisal.

\section{The Decision Problem}

The aim of the appraisal, as defined by the NICE final scope, was to assess the clinical and cost-effectiveness of FAc within its marketing authorisation for treating recurrent non-infectious uveitis. Uveitis is a sight-threatening inflammation of the components of the eye located in the uvea (iris, ciliary body, and choroid) [4]. It is a significant cause of sight loss in the working-age population, and is responsible for up to $15 \%$ of cases with legal blindness [5]. Sight loss in uveitis is most commonly due to macular oedema (fluid in the central part of the retina), glaucoma (damage to the optic nerve from high intraocular pressure), or cataract. Non-infectious uveitis affecting the anterior part of the eye only is usually easily treated with eye drops and has a low risk of sight loss. In contrast, non-infectious uveitis affecting the posterior segment of the eye (NIU-PS) is associated with a higher risk of sight loss and cannot be treated by drops alone. NIU-PS, which includes intermediate, posterior and panuveitis, was the focus of the CS.

There is no consensus clinical guidance regarding the treatment of NIU-PS in the UK. The company considered that FAc could be administered as first-, second- or thirdline treatment.

The NICE final scope listed the following comparators [6]:

- periocular or intravitreal corticosteroid injections;

- intravitreal corticosteroid implants, including dexamethasone intravitreal implant (dexamethasone);

- systemic corticosteroids;

- systemic immunosuppressive therapies;

- tumour necrosis factor (TNF)- $\alpha$ inhibitors, including adalimumab;

- best supportive care (BSC; when all other treatment options have been tried).

None of these comparators was explicitly compared with FAc in the CS since the company only provided a comparison of FAc versus (limited) current practice [(L)CP], which was the comparator arm of the pivotal trial (PSV-FAI-001).

The CS focussed on a narrower population than defined by the NICE scope, but was in line with the marketing authorisation. The place of FAc in the treatment pathway was unclear, which was problematic because the comparators of interest were dependent on the place of FAc in the treatment pathway.

\section{Independent Evidence Review Group Review}

The following sections contain a summary of the evidence submitted by the company and the critique of that evidence by the ERG.

\subsection{Clinical Effectiveness Evidence Submitted by the Company}

The company identified one relevant randomised controlled trial (RCT) of FAc and its comparators in patients with recurrent or persistent NIU-PS [1, 7]. The PSV-FAI-001 
trial (NCT01694186) was a 36-month parallel, randomised, double-blind trial, comparing FAc implant $(0.18 \mathrm{mg})$ versus (L)CP (sham injection). The study included 129 patients from six countries (USA, India, Israel, UK, Germany and Hungary), with 20 patients from the UK [16 (18.4\%) in the FAc arm and 4 (9.5\%) in the (L)CP arm]. Patients were randomised in a 2:1 ratio to the FAc and (L)CP arms of the trial.

The primary efficacy analysis was performed on the intention-to-treat (ITT) population at 6 months and compared the proportion of patients in the treatment and control groups who had a recurrence of uveitis in the study eye in the 6 months following day 1 .

For the primary endpoint ITT analysis, data on the recurrence of uveitis was imputed as follows: (1) a patient was considered as having a recurrence when he/she had not previously experienced a recurrence and missed an eye examination for the assessment of recurrence during follow-up; and (2) a patient who had not previously experienced a recurrence and took a prohibited concomitant medication (systemic treatment or local treatment of the study eye) at any time during the study follow-up prior to being considered as having a recurrence.

In terms of a recurrence of uveitis in the study eye, results showed statistically significant benefits of FAc over (L)CP at $6(27.6 \%$ vs. $90.5 \%)$ and 12 months (37.9\% vs. $97.6 \%)$. There was a clear effect in terms of time to first recurrence of uveitis in favour of FAc when compared with (L)CP. Most recurrences were imputed [ 23 of 24 and 26 of 38 in the FAc and (L)CP arms respectively at 6 months]. The results of secondary outcomes showed that more patients in the FAc arm than in the (L)CP arm experienced recurrence of uveitis in the fellow eye at 12 months $(86.4 \%$ vs. $74.2 \%)$. In terms of visual acuity in the study eye, results seemed to favour FAc over (L)CP; however, the statistical significance of the results in terms of visual acuity was not initially reported. It was therefore possible that none of these results showed a statistically significant difference. In addition, in terms of the need for further corticosteroid treatment, results favoured FAc over (L)CP, but, again, the significance of the results was not reported at the time of the submission. Healthrelated quality of life was not assessed in the PSV-FAI-001 trial.

The incidence of at least one treatment-emergent adverse event (TEAE) during 12 months of follow-up was $80 \%$ for FAc and 93\% for (L)CP study eyes. Approximately half of the participants in both treatment groups experienced a nonocular AE during the first 12 months of the study. Sixteen participants, $9(10.3 \%)$ in the FAc group and $7(16.7 \%)$ in the (L)CP group, experienced protocol-defined serious ocular AEs. The most common serious ocular AEs were increased intraocular pressure (IOP; two participants [2.3\%]) and cataract (four participants [4.6\%]) in the FAc group, and macular edema, non-infectious endophthalmitis, and uveitis (each experienced by two participants [4.8\%]) in (L)CP study eyes. Serious AEs, ocular or non-ocular, were manageable and did not result in either treatment or study discontinuation [7].

\subsection{Critique of Clinical Effectiveness Evidence and Interpretation}

The CS and response to clarification provided sufficient details for the ERG to appraise the searches for eligible studies. Searches were carried out in accordance with the NICE guide to the methods of technology appraisal Sections 5.2.2 and 5.2.4 using a good range of databases [8]. Additional searches of HTA agencies, clinical trials registries, conference proceedings and reference checking were reported.

No attempt was made to search for most comparators mentioned in the scope (periocular or intravitreal corticosteroid injections, intravitreal corticosteroid implants, systemic corticosteroids, systemic immunosuppressive therapies, and TNF $\alpha$ inhibitors). Only two active comparators were included in the literature search performed by the company-adalimumab and dexamethasone; however, the company decided not to perform an indirect comparison with these two comparators. Therefore, the only comparison presented in the CS was FAc versus (L)CP from the PSV-FAI-001 trial. The company argued that "the (L)CP arm of PSV-FAI-001 is considered largely representative of current practice in the UK for the treatment of uveitic flares and recurrence" [1]; however, the ERG did not agree with this claim [3].

The PSV-FAI-001 trial did not provide evidence for the use of FAc as first-line treatment. All patients in the trial had received treatment with systemic corticosteroid or other systemic therapies during the 12 months prior to enrolment. Therefore, the ERG believed that the most likely place of FAc in the treatment pathway was in second-line alongside dexamethasone, which made intraocular dexamethasone the most appropriate comparator. A comparison with adalimumab was relevant if the committee believed FAc was a third-line treatment option. Regarding BSC, the independent clinical expert consulted by the ERG advised that BSC (i.e. the absence of active treatment) is very rare in active disease.

Results from the PSV-FAI-001 trial showed that FAc provided significant benefits compared with (L)CP in terms of recurrence of uveitis. However, what was reported as recurrence of uveitis was largely prescription of so-called 'prohibited medication', which is not an adequate measure of this outcome. This is because its prescription may be indicated for a number of reasons other than recurrence of uveitis in the study eye, including recurrence in the fellow eye and deterioration of an underlying autoimmune condition. This was likely to have led to an underestimation of treatment effectiveness in each arm of the trial. However, it was not known how this influences the relative effectiveness of FAc 
versus $(\mathrm{L}) \mathrm{CP}$. It is also questionable whether $(\mathrm{L}) \mathrm{CP}$ was representative of UK clinical practice (particularly since the NICE approval of the dexamethasone implant), and the CS did not present any comparisons with another active treatment for NIU-PS (e.g. dexamethasone, corticosteroids or immunosuppressants). Most recurrences in the trial were imputed, which most likely led to a biased estimation of the (relative) effectiveness of FAc. The magnitude and direction of this bias are unknown.

\subsection{Cost-Effectiveness Evidence Submitted by the Company}

The company conducted three systematic reviews to identify relevant cost-effectiveness analyses, health-related qualityof-life studies, and resource use and cost studies in order to inform its assessment. One relevant resource use and cost study was identified during the review process.

A de novo Markov model, including five health states ('on treatment', 'subsequent treatment', 'remission', 'permanent blindness' and 'death'), was developed by the company (Fig. 1). This model structure was informed by a previous technology appraisal (adalimumab and dexamethasone for treating non-infectious uveitis, TA 460 [9]) and only considered the treated eye of patients during the PSV-FAI-001 trial. Patients entered the model in the 'on treatment' health state, and transited to the 'subsequent treatment' health state upon recurrence. It was assumed that patients could only transit to the 'permanent blindness' health state after treatment failure.
Patients were considered in 'remission' when they did not experience disease recurrence for a period of two years.

The cost-effectiveness analysis considered FAc versus (L)CP for treating recurrent or persistent NIU-PS, which was in line with the marketing authorisation. It was assumed that a single FAc implant was implanted at the start of the model. Supplemental treatments (i.e. the treatments that were tapered off at the beginning of the trial) were considered during the first 12 weeks of the model only, which meant that patients in the (L)CP arm were assumed not to receive any active treatment for NIU-PS after approximately 3 months until recurrence.

The model adopted an NHS and Personal and Social Services (PSS) perspective in England and Wales. The cycle length was 2 weeks and the time horizon was 51 years. A half-cycle correction was not applied. All costs and qualityadjusted life-years (QALYs) were discounted at a rate of $3.5 \%$ per year.

The proportion of patients 'on treatment' was informed by the time to first recurrence in the treated eye observed in the PSV-FAI-001 trial, and the remaining transition probabilities were informed by the literature. The Kaplan-Meier (KM) curves representing time to first recurrence of the PSV-FAI-001 trial were digitised to inform the proportion of patients 'on treatment' over time. In the FAc arm, a piecewise model was used to model time to first recurrence. The first 120 days of the piecewise model were directly informed by the KM curve and then by a parametric time-to-event model fitted to the remainder of the KM curve. This was justified by the identification of a 'drop' in the KM curve

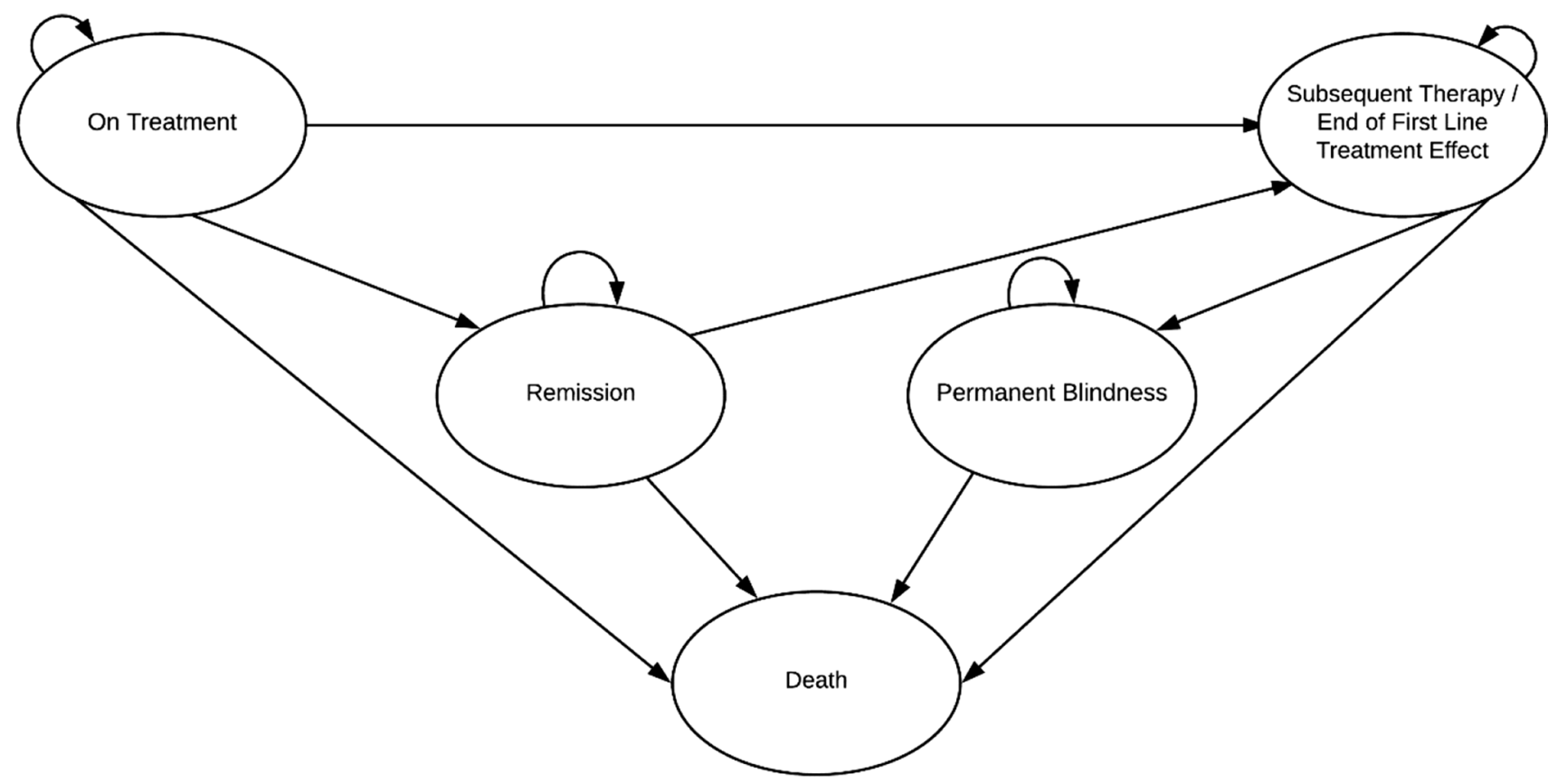

Fig. 1 Model structure. Source: company submission [1] 
representing time to first recurrence in the FAc arm. In the (L)CP arm, time to first recurrence was modelled through a parametric time-to-event model fitted to the entire KM curve. Multiple distributions (exponential, Weibull, loglogistic, lognormal, gamma, Gompertz, generalised gamma, and generalised F) were fitted to KM curves. The exponential distribution was selected for the FAc arm after 120 days and the log-logistic distribution was selected for the (L)CP arm, based on statistical fit (both distributions had the best statistical fit) and visual inspection. Dick et al. informed the transition from 'subsequent therapy' to 'permanent blindness'[10]. The transition to 'death' from all health states was equal to UK general population mortality probabilities.

Treatment-related adverse events occurring in at least 5\% of patients in either treatment arm of PSV-FAI-001 were included in the 'on treatment' and 'remission' health states of the cost-effectiveness model.

Quality-of-life data were not measured in the PSVFAI-001 trial and no relevant studies were identified during the systematic literature review. Hence, the 'on treatment' and 'subsequent treatment' health state utility values were obtained through mapping. The company used a mapping algorithm that maps the Visual Function Questionnaire (VFQ)-25 data into EuroQol-5 dimensions (EQ-5D) data. This mapping algorithm was developed during TA460 based on the HURON trial [9]. In the current appraisal, this mapping algorithm was applied to the VFQ-25 data reported in the MUST trial [11] to obtain the 'on treatment' and 'subsequent treatment' health state utility values. The utility value associated with 'permanent blindness' (0.38) was taken from the literature [12]. Patients in the 'remission' health state were assumed to have utility values equal to the age-matched general UK population utility values [13]. No utility decrements were associated with the occurrence of adverse events.

The following cost categories were included in the costeffectiveness model: acquisition and administration costs of the intervention, monitoring costs, costs of supplemental and subsequent treatments, costs of permanent blindness, and costs of managing adverse events. The list price of a single FAc implant was $£ 5500$, but the company presented results based on a patient access scheme (PAS) price. The 'permanent blindness' costs were divided in one-off transition costs, as well as cyclic costs. The transition costs contained the costs of registration as a blind person, the costs of low vision aids, low vision rehabilitation, and the costs of residential care, while the cyclic costs contained the monitoring costs and the costs of depression, hip replacement and community care.

FAc was associated with higher QALY gains and higher costs than (L)CP. The probabilistic incremental cost-effectiveness ratio (ICER; 1000 iterations) of FAc versus (L)CP was $£ 7702$ per QALY gained. Based on the deterministic sensitivity analyses (DSA), the most influential parameters on the cost-effectiveness results were the 'subsequent treatment' and 'on treatment' health state utility values, and the 45-54 years age-matched utility value that informed the 'remission' health state utility value.

\subsection{Critique of the Cost-Effectiveness Evidence and Interpretation}

Literature searches were well-documented, reproducible, and performed according to the NICE requirements.

The main shortcomings of the company's model structure were that it did not capture the effect of treatment on patients' visual acuity, and that the company modelled the impact of NIU-PS and treatment in the treated eye only. The ERG considered this approach inappropriate because patients were at risk of developing bilateral disease. Hence, including the consequences of the disease and treatment in both eyes would have been more appropriate. The use of a 'remission' health state and the lack of transition between the 'on treatment' and 'permanent blindness' health states were not considered to be appropriate by the ERG.

The company's cost-effectiveness model considered a single FAc implant. The ERG believed that clinicians may consider implanting subsequent implants in patients who would respond to a first implant. The ERG further wondered about the representativeness of (L)CP for UK clinical practice, and critiqued the lack of comparison with dexamethasone and other comparators listed in the NICE final scope. Subgroup analyses mentioned in the scope were not performed.

The estimation of time to first recurrence in the costeffectiveness model suffered from the limitations described under Sect. 3.2. Additionally, the company decided to use a piecewise model to model time to first recurrence in the FAc arm because of the 'drop' identified in the KM curve representing time to first recurrence. However, the company acknowledged that this 'drop' was likely caused by the design of the PSV-FAI-001 trial and not by the influence of treatment on the time to first recurrence; one could wonder whether this 'drop' was representative of UK clinical practice. Uncertainty therefore remains regarding the effectiveness of FAc on time to first recurrence. Furthermore, the company used different approaches to model time to first recurrence in the FAc and (L)CP arms, a piecewise model, and a standard parametric time-to-event model respectively. This may have influenced the outcomes irrespective of differences in treatment effectiveness between FAc and (L)CP. The size and magnitude of the bias introduced by all these assumptions could not be quantified by the ERG. Additional uncertainties were introduced by the lack of evidence regarding the effectiveness of FAc after 3 years, i.e. when the implant is no longer releasing any active substance, and 
the use of digitised KM curves instead of individual patientlevel data.

Quality of life remained uncertain because it was not measured during the PSV-FAI-001 trial. Utility values were calculated by applying a mapping algorithm, developed based on data from the HURON trial, to the MUST VFQ-25 data $[9,11]$. The populations in these trials differed from each other and from the population of interest in this appraisal, hence the utility estimates may be biased and their relevance for the current appraisal compromised. Utility decrements for adverse events were not included in the company's analyses and utility values were not capped to UK general population estimates. Additionally, the 'remission' health state utility values were assumed to be equal to UK general population utility values, which were considered to be unrealistic by the ERG. The resulting total QALYs estimated by the company in both treatment arms were likely overestimated.

The estimation of the 'permanent blindness' health state costs was not considered appropriate because these were obtained from a macular degeneration population and contained the costs of hip replacement, community care, and residential care. These cost items were not considered by the ERG to be relevant for the NIU-PS population. The ERG further identified multiple errors in the implementation of the cost calculations in the company's cost-effectiveness model.

The probabilistic sensitivity analysis (PSA) did not contain the rate of blindness and almost all parameters were varied by $10 \%$ around the mean. This led to an underestimation of the uncertainty surrounding the results. The ERG further noticed that the scenarios presented by the company did not explore the impact of all structural assumptions on the results.

The ERG was concerned about the quality of the validation efforts performed by the company since multiple implementation errors were identified in the company's costeffectiveness model. Furthermore, the company's experts' opinion elicitation and the validation efforts using data from TA460 were not transparently reported.

\subsection{Additional Work Undertaken by the ERG}

Based on the above-described critiques, the ERG amended the company's cost-effectiveness model. These amendments involved fixing errors and including dexamethasone as a comparator (fixing violation) [14]. Since an indirect comparison of FAc versus dexamethasone could not be performed, the ERG presented three sets of scenario analyses assuming a different effectiveness of dexamethasone. In the first set of analyses, the hazard ratio of dexamethasone versus (L) $\mathrm{CP}$ was assumed to be 0.456 . This hazard ratio was calculated based on the results of TA460, which compared (L)CP with dexamethasone. To obtain this hazard ratio, the ERG reproduced the incremental QALY gained of 0.029 that was observed in TA460. This estimation rests on the assumption that the incremental QALY gained of dexamethasone versus (L)CP is equal in both appraisals, regardless of the differences in structural assumptions, which was acknowledged to be a strong assumption by the ERG. In the second set of analyses, the same effectiveness was assumed between dexamethasone and FAc (hazard ratio of 1). In the third set of analyses, dexamethasone was assumed to be more effective than FAc (hazard ratio of 0.7 for dexamethasone vs. FAc). All hazard ratios were applied to the parametric time-toevent models representing time to first recurrence.

The following additional assumptions were made to incorporate dexamethasone in the cost-effectiveness model: dexamethasone was considered to be effective for 6 months (i.e. a new dexamethasone implant was assumed to be implanted every 6 months), multiple dexamethasone implants were administered to equal the active treatment period of FAc, the acquisition cost of dexamethasone was $£ 870$ and its administration cost was $£ 113.42$. The remaining model inputs and assumptions of dexamethasone were assumed to be the same as for FAc.

The ERG used the individual patient data instead of the digitised KM curve to estimate time to first recurrence, capped utility values to UK general population values, assumed equal supplemental treatment costs between FAc and (L)CP, corrected the doses of subsequent and supplemental treatments, and used the standard errors instead of $10 \%$ variation around the mean for the parameters included in the PSA, when available (fixing violations). Multiple matters of judgement were also incorporated in the ERG's preferred analyses: removing the 'remission' health state, including a transition from the 'on treatment' to the 'permanent blindness' health state, assuming the same probability of recurrence after 3 years in the FAc as in the (L)CP arm, adjusting the 'permanent blindness' health state costs, adding blood tests in the 'subsequent treatment' health state, and assuming the same transition costs to the 'subsequent treatment' health state after 3 years.

All these amendments were included in the ERG basecase 1 analysis. Three additional ERG base-case analyses were defined. ERG base-case 2 included a utility decrement of 0.05 for all adverse events. ERG base-case 3 assumed that patients could receive multiple FAc implants [the probability of recurrence in the FAc arm after 3 years was then not assumed equal to the probability of recurrence in the (L)CP arm]. ERG base-case 4 included both the utility decrements for adverse events and the possibility of receiving multiple FAc implants. Because the model did not allow for a PSA including three treatments, all ERG results were presented deterministically. 
FAc extendedly dominated dexamethasone when a hazard of 0.456 was assumed for dexamethasone versus (L) CP. Dexamethasone dominated FAc when assuming equal effectiveness and FAc was extendedly dominated by dexamethasone when assuming a hazard ratio of 0.7 for dexamethasone versus FAc. In all ERG base-case analyses, the ICERs of FAc versus (L)CP remained under $£ 31,000$ per QALY gained (Table 1).

Additional exploratory analyses were performed by the ERG. The most influential assumptions, all leading to ICERs above $£ 20,000$ per QALY gained, were using alternative health state utility values for the 'on treatment' and 'subsequent treatment' health state, including higher utility decrements for adverse events $(-0.1)$, and assuming no treatment effectiveness of dexamethasone and FAc after 3 years.

\subsection{Conclusions of the ERG Report}

The ERG believed that the effectiveness and cost-effectiveness of FAc were uncertain because of (1) the lack of evidence regarding any relevant comparator; (2) the primary outcome of the trial, 'recurrence of uveitis', was not adequately assessed and the vast majority of recurrences were imputed; and (3) the unavailability of quality-of-life data elicited in the population of interest.

\subsection{Company Response to the First Appraisal Committee Meeting and ERG Critique}

In response to the first $\mathrm{AC}$ meeting, after which a 'minded no' recommendation was issued, the company provided additional cost-effectiveness analyses that included some of the ERG's preferred assumptions and considered dexamethasone as a comparator. The ERG and the company analyses differed in how dexamethasone was implemented in the costeffectiveness model. In its response, the company assumed that dexamethasone and FAc were equally effective for their respective active period. The active period of FAc was considered to be 3 years and the active period of dexamethasone was assumed to be 6 months.

In the company's analyses, the effectiveness of FAc was 'scaled down' from 3 years to 6 months to represent the effectiveness of dexamethasone. Hence, the proportion of patients 'on treatment' after 6 months in the dexamethasone arm was the same as the proportion of patients 'on treatment' after 3 years in the FAc arm. The company assumed that a subsequent implant was directly administered after treatment failure instead of after the end of the 'active' period of the implants, i.e. 6 months for dexamethasone and 3 years for FAc. The company recalculated the utility decrements associated with adverse events based on additional information regarding the occurrence of adverse events. The additional analyses of the company contained the following comparisons:

- FAc implant versus dexamethasone implant (one, two and three implants of each);

- one FAc implant versus one, two and three dexamethasone implants;

- one dexamethasone implant followed by one FAc implant versus two and three dexamethasone implants.

The company response also contained results for bilateral disease. These were obtained by doubling the results obtained from the cost-effectiveness model. Finally, a comparison of visual acuity in the FAc and (L)CP arms at different time points was provided, using a one-way analysis of variance (ANOVA).

The main critique of the ERG on these additional analyses was that the company did not assume equal effectiveness between FAc and dexamethasone because patients in the dexamethasone arm did not respond to treatment approximately six times faster than patients in the FAc arm. Additionally, the ERG wondered about the relevance of the provided analyses because the time on treatment of one, two, or three dexamethasone implants is by definition shorter than one, two, or three FAc implants; hence, the FAc arm would automatically lead to higher health benefits. Comparing one FAc implant with six dexamethasone implants, as performed in the ERG analyses, seemed more appropriate. Doubling the results of the cost-effectiveness model to obtain results for bilateral disease did not capture the effect of treatment on visual acuity in both eyes, and consequently on quality of life and costs. The reliability of the ANOVA to compare visual acuity results seemed questionable to the ERG because the visual acuity data did not seem to be normally distributed.

\section{Key Methodological Issues}

It was unclear whether FAc would be administered as a first, second, or third treatment line in the treatment pathway. This element of the scope was not clearly defined but was important for the decision to be taken because it determined which comparator was relevant. Additionally, it was unclear whether subsequent FAc implants would be considered. This ambiguity emphasises that it is of utmost importance to accurately define the scope of an appraisal in order for the company and the ERG to deliver the most informative evidence and analyses.

Another major issue was whether the model structure adequately represented the natural course of the disease since it did not capture the effect of the disease and treatment on visual acuity. The relation between health states, visual 
Table 1 Deterministic company and ERG base-case results

\begin{tabular}{|c|c|c|c|c|}
\hline Assumed effectiveness of DEX & Analysis & Comparator & $\begin{array}{l}\text { Fully incremental ICER (incre- } \\
\text { mental £/QALY) }\end{array}$ & $\begin{array}{l}\text { ICER of FAc } \\
\text { versus com- } \\
\text { parator }\end{array}$ \\
\hline DEX not considered & Company base-case & (L)CP & - & $£ 7183$ \\
\hline \multirow[t]{12}{*}{ HR of 0.456 for DEX versus (L)CP } & \multirow[t]{3}{*}{ ERG base-case 1} & (L)CP & Cheapest & $£ 12,325$ \\
\hline & & DEX & Extendedly dominated & $£ 5335$ \\
\hline & & FAc & $£ 12,325$ & - \\
\hline & \multirow[t]{3}{*}{ ERG base-case 2} & $(\mathrm{~L}) \mathrm{CP}$ & Cheapest & $£ 21,531$ \\
\hline & & DEX & Extendedly dominated & $£ 9457$ \\
\hline & & FAc & $£ 21,531$ & - \\
\hline & \multirow[t]{3}{*}{ ERG base-case 3} & (L)CP & Cheapest & $£ 19,049$ \\
\hline & & DEX & Extendedly dominated & $£ 13,856$ \\
\hline & & FAc & $£ 19,049$ & - \\
\hline & \multirow[t]{3}{*}{ ERG base-case 4} & $(\mathrm{~L}) \mathrm{CP}$ & Cheapest & $£ 30,153$ \\
\hline & & DEX & Extendedly dominated & $£ 22,810$ \\
\hline & & FAc & $£ 30,153$ & - \\
\hline \multirow{12}{*}{$\begin{array}{l}\text { HR of } 1 \text { for DEX versus FAc (i.e. same } \\
\text { effectiveness) }\end{array}$} & \multirow[t]{3}{*}{ ERG base-case 1} & $(\mathrm{~L}) \mathrm{CP}$ & Cheapest & $£ 12,325$ \\
\hline & & DEX & $£ 12,283$ & Dominated \\
\hline & & FAc & Dominated & - \\
\hline & \multirow[t]{3}{*}{ ERG base-case 2} & $(\mathrm{~L}) \mathrm{CP}$ & Cheapest & $£ 21,531$ \\
\hline & & DEX & $£ 21,457$ & Dominated \\
\hline & & FAc & Dominated & - \\
\hline & \multirow[t]{3}{*}{ ERG base-case 3} & (L)CP & Cheapest & $£ 19,049$ \\
\hline & & DEX & $£ 18,710$ & Dominated \\
\hline & & FAc & Dominated & - \\
\hline & \multirow[t]{3}{*}{ ERG base-case 4} & (L)CP & Cheapest & $£ 30,153$ \\
\hline & & DEX & $£ 29,617$ & Dominated \\
\hline & & FAc & Dominated & - \\
\hline \multirow[t]{12}{*}{ HR of 0.7 for DEX versus FAc } & \multirow[t]{3}{*}{ ERG base-case 1} & $(\mathrm{~L}) \mathrm{CP}$ & Cheapest & $£ 12,325$ \\
\hline & & FAc & Extendedly dominated & - \\
\hline & & DEX & $£ 10,412$ & $£ 2297$ \\
\hline & \multirow[t]{3}{*}{ ERG base-case 2} & $(\mathrm{~L}) \mathrm{CP}$ & Cheapest & $£ 21,531$ \\
\hline & & FAc & Extendedly dominated & - \\
\hline & & DEX & $£ 17,843$ & $£ 3643$ \\
\hline & \multirow[t]{3}{*}{ ERG base-case 3} & (L)CP & Cheapest & $£ 19,049$ \\
\hline & & FAc & Extendedly dominated & - \\
\hline & & DEX & $£ 17,239$ & $£ 12,911$ \\
\hline & \multirow[t]{3}{*}{ ERG base-case 4} & $(\mathrm{~L}) \mathrm{CP}$ & Cheapest & $£ 30,153$ \\
\hline & & FAc & Extendedly dominated & - \\
\hline & & DEX & $£ 25,074$ & $£ 15,730$ \\
\hline
\end{tabular}

Sources: Tables 5.5 and 5.11-5.13 of the ERG report [3]

$D E X$ dexamethasone, ERG Evidence Review Group, FAc fluocinolone acetonide, $H R$ hazard ratio, ICER incremental cost-effectiveness ratio, $(L)$ $C P$ (limited) clinical practice, $Q A L Y$ quality-adjusted life-year

acuity, and their associated utility values was thus unclear. Additionally, the company included the consequences of treatment in the treated eye only, while NIU-PS is a disease that can develop into bilateral disease. Neglecting the impact of the disease in the second eye of patients therefore biases the estimation of health benefits and costs.
The company's cost-effectiveness analyses were highly uncertain because of the multiple shortcomings and uncertainties identified in the evidence submitted by the company (e.g. lack of evidence regarding any relevant comparator, uncertainty surrounding the model structure, and the unavailability of quality-of-life data). The impact of these uncertainties on the results was extensively explored by the ERG 
through scenario analyses, and several of these scenarios led to requests for further analyses by the AC. This appraisal therefore shows that scenarios are powerful analyses to support decision making in case of sparse evidence.

\section{National Institute for Health and Care Excellence Guidance}

NICE recommended the FAc within its marketing authorisation for treating recurrent non-infectious uveitis on the 20th of June 2019. This section summarises the committee discussion; full detail can be found in the FAD [2].

\subsection{Intervention}

The AC understood from patient experts that having a longlasting treatment ( 3 years) would increase patients' quality of life since the treatment of recurrent uveitis requires frequent hospital visits, which may disrupt patients' daily life; they also noted that systemic treatments may be relatively contraindicated for some patients. Clinical experts advised that $20-30 \%$ of patients do not respond to currently available biologic treatments. For these reasons, a new long-lasting treatment option was deemed valuable.

\subsection{Comparator}

Clinical experts explained that they would consider administering a FAc implant instead of a subsequent dexamethasone implant to patients who originally responded to a dexamethasone implant. Based on this explanation of the potential treatment pathway, the AC deemed that dexamethasone was a relevant comparator for FAc.

\subsection{Considerations of Clinical Effectiveness}

The AC concluded that the PSV-FAI-001 trial may not represent UK clinical practice because patients in the $(\mathrm{L}) \mathrm{CP}$ arm of the trial did not receive treatment after 3 months and before recurrence, and because investigators were encouraged to first treat recurrence of uveitis with local and then systemic treatment (upon failure of local treatments). This was not considered to represent UK practice because systemic treatment may be administered directly in case of bilateral or systemic disease.

The AC established that the recurrence rate of uveitis was overestimated because recurrences were imputed when patients missed an eye examination, or were treated with local or systemic treatments. The trial did not register why these treatments were administered but the AC noticed that these treatments may have been used to treat the non-study eye or an underlying condition.

The AC recognised that FAc improved visual acuity based on the improvement in best-corrected visual acuity provided by FAc compared with (L)CP. The AC noted that the cost-effectiveness model did not capture the effect of treatment on visual acuity.

Cataract and IOP are common adverse events associated with FAc. Clinical experts expected the toxicity profile of FAc to be similar to the toxicity profile of dexamethasone and explained that the adverse events associated with FAc would not lead to a long-term decrease in quality of life. The AC concluded that the adverse events profile of FAc was well tolerated and manageable.

\subsection{Considerations of Cost-Effectiveness}

The model structure of the company only considered the treated eye during the trial. However, clinical experts advised that a large proportion of patients may have bilateral disease (either from outset or becoming bilateral during the course of disease); $67.8 \%$ of patients in the FAc arm and $73.8 \%$ patients in the control arm had bilateral disease at the start of the PSV-FAI-001 trial. Therefore, the AC concluded that a cost-effectiveness model considering both eyes would have been more suitable. Additionally, the AC concluded that the cost-effectiveness model should not include a 'remission' health state and that the results both with and without transition from the 'on treatment' to 'permanent blindness' health state were relevant.

The AC concluded that multiple implants should be considered in the cost-effectiveness model because clinical experts stated that they would consider implanting a subsequent FAc implant in patients who responded to a first implant.

The AC established that dexamethasone was a relevant comparator for FAc. During its deliberations, the AC considered both the ERG and company's methods of implementing dexamethasone in the cost-effectiveness model. The AC heard that dexamethasone, over a period of 3 years (six dexamethasone implants), was expected to be slightly less effective than FAc. The AC concluded that the ERG implementation of dexamethasone was more plausible than the company's implementation of dexamethasone because this later led to an implausible relative effectiveness of dexamethasone and to different treatment durations between the dexamethasone and FAc arms.

Regarding the model inputs, the AC concluded that the cost-effectiveness model should not contain treatment benefits of FAc after 3 years. The AC noted that there was some uncertainty surrounding the methods used to estimate utility decrements associated with AEs in the company's response 
to the first AC meeting, but the AC concluded that the company's method provided more reliable estimates than the ERG method. Finally, the AC stated that the ERG changes regarding the resource use and costs calculated were plausible but had little effects on the cost-effectiveness results.

The AC considered FAc to be a cost-effective use of NHS resources, had all its preferred assumptions been included in the cost-effectiveness model.

\section{Conclusions}

This paper summarises the STA of FAc for treating recurrent non-infectious uveitis. The ERG identified multiple shortcomings in the evidence submitted by the company, such as the lack of evidence regarding any relevant comparator, the likely biased treatment effectiveness estimations, the model structure not capturing the effect of treatment on visual acuity, and the unavailability of quality-of-life data in the population of interest. Even though the AC recognised these gaps in the evidence base, it recommended FAc for the treatment of NIU-PS because the AC considered FAc to be a cost-effective use of NHS resources, had all its preferred assumptions been included in the analysis.

Acknowledgements This summary of the ERG report was compiled after NICE issued the FAD. All authors have commented on the submitted manuscript and have given their approval for the final version to be published. The views and opinions expressed therein are those of the authors and do not necessarily reflect those of NICE or the Department of Health. Any errors are the responsibility of the authors.

Author contributions All authors have commented on the submitted manuscript and have given their approval for the final version to be published. RR, AC, VHC, HR and JK reviewed the clinical-effectiveness evidence; $\mathrm{CN}$ reviewed the search methods. GW acted as statistician and critiqued the analyses in the company's submission. AD contributed to the writing of the report and provided clinical advice. XP, SP, WW, NA, DS and MJ reviewed the cost-effectiveness evidence. $\mathrm{XP}$ acts as overall guarantor for the manuscript. This summary has not been externally peer reviewed by PharmacoEconomics

\section{Compliance with Ethical Standards}

Funding This project was funded by the National Institute for Health Research (NIHR) Health Technology Assessment (HTA) Programme (Project number 16/119/02). Visit the HTA programme website for further project information (https://www.nihr.ac.uk/explore-nihr/fundi ng-programmes/health-technology-assessment.htm).

Conflict of interest Xavier G. L. V. Pouwels, Svenja Petersohn, Vanesa Huertas Carrera, Alastair K. Denniston, Annette Chalker, Heike Raatz, Nigel Armstrong, Dhwani Shah, Willem Witlox, Gill. Worthy, Caro Noake, Rob Riemsma, Jos Kleijnen, and Manuela A. Joore have no conflicts of interest to declare.

Open Access This article is distributed under the terms of the Creative Commons Attribution-NonCommercial 4.0 International License (http://creativecommons.org/licenses/by-nc/4.0/), which permits any noncommercial use, distribution, and reproduction in any medium, provided you give appropriate credit to the original author(s) and the source, provide a link to the Creative Commons license, and indicate if changes were made.

\section{References}

1. Alimera Sciences. Fluocinolone acetonide ocular implant for treating recurrent non-infectious uveitis [ID1039]. Document B: submission to National Institute of Health and Care Excellence. Single technology appraisal (STA): Alimera Sciences. 2018. https ://www.nice.org.uk/guidance/ta590/documents/committee-paper s. Accessed 27 Nov 2018.

2. National Institute for Health and Care Excellence. Fluocinolone acetonide intravitreal implant for treating recurrent non-infectious uveitis: final appraisal document. London: NICE; 2019. https:// www.nice.org.uk/guidance/gid-ta10368/documents/final-appra isal-determination-document. Accessed 11 July 2019.

3. Riemsma R, Pouwels X, Petersohn S, Chalker A, Huertas Carrera V, Raatz H, et al. Fluocinolone acetonide ocular implant for treating recurrent non-infectious uveitis: a single technology assessment. York: Kleijnen Systematic Reviews Ltd; 2019. https ://www.nice.org.uk/guidance/ta590/documents/committee-paper s. Accessed 11 July 2019.

4. National Eye Institute. Facts about uveitis. 2011. https://nei.nih. gov/health/uveitis/uveitis. Accessed 29 Nov 2018.

5. Chen SC, Sheu SJ. Recent advances in managing and understanding uveitis. F1000Res. 2017;6:280.

6. National Institute for Health and Care Excellence. Health technology appraisal: fluocinolone acetonide ocular implant for treating recurrent non-infectious uveitis: final scope. London: NICE; 2018. https://www.nice.org.uk/guidance/ta590/documents/final-scope. Accessed 28 Sept 2018.

7. Jaffe GJ, Foster S, Pavesio C, Paggiarino D, Riedel GE. Effect of an injectable fluocinolone acetonide insert on recurrence rates in noninfectious uveitis affecting the posterior segment: 12-month results. Ophthalmology. 2019;126(4):601-10.

8. National Institute for Health and Care Excellence. Guide to the methods of technology appraisal 2013. London: NICE; 2013. https://www.nice.org.uk/process/pmg9/resources/guide-to-themethods-of-technology-appraisal-2013-pdf-2007975843781. Accessed 28 May 2013.

9. National Institute for Health and Care Excellence. Adalimumab and dexamethasone for treating non-infectious uveitis. NICE technology appraisal guidance 460. London: NICE; 2017. https ://www.nice.org.uk/guidance/ta460. Accessed 5 Oct 2018.

10. Dick AD, Tundia N, Sorg R, Zhao C, Chao J, Joshi A, et al. Risk of ocular complications in patients with noninfectious intermediate uveitis, posterior uveitis, or panuveitis. Ophthalmology. 2016;123(3):655-62.

11. Multicenter Uveitis Steroid Treatment Trial Follow-up Study Research Group. Quality of life and risks associated with systemic anti-inflammatory therapy versus fluocinolone acetonide intraocular implant for intermediate uveitis, posterior uveitis, or panuveitis: fifty-four-month results of the multicenter uveitis steroid treatment trial and follow-up study. Ophthalmology. 2015;122(10):1976-86.

12. Czoski-Murray C, Carlton J, Brazier J, Young T, Papo NL, Kang HK. Valuing condition-specific health states using simulation contact lenses. Value Health. 2009;12(5):793-9.

13. Janssen B, Szende A. Population norms for the EQ-5D. In: Janssen B, Szende A, Cabases J, editors. Self-reported population 
health: an international perspective based on EQ-5D. Dordrecht: Springer; 2013. p. 19-30.

14. Kaltenthaler E, Carroll C, Hill-McManus D, Scope A, Holmes M, Rice $\mathrm{S}$, et al. The use of exploratory analyses within the National
Institute for Health and Care Excellence single technology appraisal process: an evaluation and qualitative analysis. Health Technol Assess. 2016;20(26):1-48.

\section{Affiliations}

Xavier G. L. V. Pouwels ${ }^{1}\left(\mathbb{0} \cdot\right.$ Svenja Petersohn ${ }^{1} \cdot$ Vanesa Huertas Carrera $^{2}$ - Alastair K. Denniston ${ }^{3,4} \cdot$ Annette Chalker $^{2}$. Heike Raatz ${ }^{2,5} \cdot$ Nigel Armstrong $^{2}$. Dhwani Shah ${ }^{2}$. Willem Witlox ${ }^{1} \cdot$ Gill Worthy ${ }^{2}$. Caro Noake ${ }^{2} \cdot$ Rob Riemsma ${ }^{2}$. Jos Kleijnen ${ }^{2,6} \cdot$ Manuela A. Joore ${ }^{1,6}$

$\triangle$ Xavier G. L. V. Pouwels

xavier.pouwels@mumc.nl

1 Maastricht University Medical Centre+, Maastricht, The Netherlands

2 Kleijnen Systematic Reviews Ltd, York, UK

3 University Hospitals Birmingham NHS Foundation Trust, Birmingham, UK

4 University of Birmingham, Birmingham, UK
5 European Centre of Pharmaceutical Medicine, Basel University, Basel, Switzerland

6 Care and Public Health Research Institute (CAPHRI), Maastricht University, Maastricht, The Netherlands 